\title{
Connection System for Small and Medium-Size Wind Generators through the Integration in an MMC and NLC Modulation
}

\author{
Fernando Martinez-Rodrigo ${ }^{1, *(\mathbb{D})}$, Dionisio Ramirez ${ }^{2}\left(\mathbb{D}\right.$, Santiago de Pablo ${ }^{1}$ and Luis Carlos Herrero-de Lucas ${ }^{1} \mathbb{D}$ \\ 1 Department of Electronics Technology, University of Valladolid, 47011 Valladolid, Spain; \\ sanpab@eii.uva.es (S.d.P.); lcherrero@eii.uva.es (L.C.H.-d.L.) \\ 2 Centro de Electrónica Industrial (CEI), 28006 Madrid, Spain; dionisio.ramirez@upm.es \\ * Correspondence: fernando.martinez@uva.es; Tel.: +34-983-423-921
}

Citation: Martinez-Rodrigo, F.;

Ramirez, D.; de Pablo, S.; Herrero-de Lucas, L.C. Connection System for Small and Medium-Size Wind Generators through the Integration in an MMC and NLC Modulation. Energies 2021, 14, 2681. https:// doi.org/10.3390/en14092681

Academic Editor: Ricardo J. Bessa

Received: 5 March 2021

Accepted: 2 May 2021

Published: 7 May 2021

Publisher's Note: MDPI stays neutral with regard to jurisdictional claims in published maps and institutional affiliations.

Copyright: (c) 2021 by the authors. Licensee MDPI, Basel, Switzerland. This article is an open access article distributed under the terms and conditions of the Creative Commons Attribution (CC BY) license (https:/ / creativecommons.org/licenses/by/ $4.0 /)$.

\begin{abstract}
This paper presents a new way of organizing a wind farm with a large number of small to medium-sized turbines. Each wind generator has been included in a switching module of a modular multilevel converter (MMC), which generates the output voltage by near level control (NLC). The proposed topology reduces the number of semiconductors required, switching losses, and voltage filtering requirements. This topology replaces the usual configuration where each wind turbine is connected to a three-phase two-level back-to-back converter plus a filter and then connected in parallel with the other wind generators. To test the topology and its control performance, a case has been developed and simulated for generator configurations producing the same power, for generation imbalances between phases and for imbalances between arms. The analysis of the data shows that the converter works correctly and that it can deliver power to the grid in a balanced way even if the generation has imbalances. The generation imbalances between phases are compensated through the average value of the circulating current, while the imbalances between arms are compensated through the $50 \mathrm{~Hz}$ circulating current.
\end{abstract}

Keywords: modular multilevel converter (MMC); near level control (NLC); small wind turbine; wind farm

\section{Introduction}

Modular multilevel converters (MMCs) were first used in high voltage applications, then in medium voltage, and lately in low voltage. In high voltage applications, they are used in high voltage direct current (HVDC) transmission [1-3], including the connection of offshore wind farms to the onshore grid. In medium voltage applications, they have been used in static synchronous compensator (STATCOM) [4,5] and motor/generator drivers [6].

Small- to medium-sized wind generators are connected to the grid via two-level threephase back-to-back converters [7]. The generator-side inverter is responsible for tracking the maximum power point (MPPT) [8,9], while the second converter is connected to the grid through a filter to reduce harmonics due to pulse width modulation (PWM). When the number of wind turbines is high, they are connected in parallel after the filter and before the low or medium voltage grid connection transformer.

The system proposed in this paper places each wind generator inside a switching module (SM) of the MMC. As in any MMC, if the number of SMs per arm is small, the output voltage is obtained by multilevel PWM [10] because the number of output voltage levels is low. However, if the number of generators is high (larger number of voltage levels) it is better to use near level control (NLC) [11], because its switching losses are much lower. Therefore, when the number of generators is small (e.g., 30) it is more appropriate to use PWM, while if the number of generators is high (e.g., 60) it is better to use NLC. The second case is the one analyzed in this article. 
Some authors have presented the integration of battery energy storage (BES) in the SMs of an MMC [12-14], mainly for medium voltage applications. These works are intended to solve the problems of having a very large number of battery elements in series when two- or three-level converters are used. The mentioned problems are mainly imbalances between battery modules and reduced reliability because the failure of one battery module can render the entire battery unusable. The integration of batteries into MMC SMs can solve both problems. The use of control systems makes it possible to eliminate imbalances that may appear between phases and arms, and to operate when the grid is unbalanced [13]. The work presented in [12] studies the case where some SMs include batteries and others do not. A slightly different application is the integration of the storage batteries of a group of electric vehicles in an MMC; a control that takes into account the different charging situations of the vehicles has been proposed in [15].

Integration of solar panels into the MMC structure, by including a panel or a group of panels in each SM, can be found in the literature. In [16], DC/DC converters have been used to connect the solar panel to the SM, which carries out the MPPT and makes it possible to eliminate the grid-connection transformer. In [17], the application to a multi-megawatt photovoltaic plant, using SMs with full-bridge topology, is studied, and MPPT is performed. In [18], a solar panel is included in each SM, and a control system is developed to perform the MPPT without the need to use a DC/DC converter in each SM; also, a redundant module is added in each arm to balance the voltages of each phase.

MMCs that include solar panels in some SMs and batteries in other SMs have been proposed to reduce the variations of the generated power due to solar radiation variations. In [19], both solar panels and batteries are connected to the SM by isolated DC/DC converters.

The integration of decentralized energy resources (DER) in an MMC is analyzed in [20]. The authors noted the need to transfer power between phases and arms as a result of generation differences and proposed using regulators to balance power generation.

As stated above, it is possible to find works in the technical literature in which batteries, solar panels, or both have been included within the MMC topology. Some of them analyze how to handle the power imbalances between phases and between arms by using regulators responsible for delivering the same power through each of the phases even though the generation is unbalanced. These regulators are difficult to apply to this case since they have been developed for PWM, not for NLC; they are based on generating a voltage difference between the upper and lower arms, and, in the case of NLC, that causes large ripples of circulating current due to the low switching frequency.

This paper proposes a topology that integrates wind generators in an MMC with NLC, which is especially suitable for wind farms with a high number of generators. The paper includes a theoretical analysis of the power transfer between phases and arms. Using simulation, it is shown that it is possible to find parameter values that allow a correct operation without the need for regulators to transfer power between phases and arms and without a large increase in the arm currents.

The proposed topology has advantages, such as the use of fewer semiconductors, lower switching losses, and reduced filtering requirements. The number of semiconductors per wind generator is reduced from 12 to 8 IGBTs by replacing the grid-side converter with the half-bridge (HB) of the SM. The use of NLC instead of PWM reduces the switching frequency of the semiconductors and their power losses. In the classical wind farm configuration, the converter of each generator is connected to the grid through a filter (L or LCL), so the same number of filters is needed as the number of generators; however, in the developed topology, a single small filter is needed, due to the multilevel voltage, which can even be eliminated in many cases.

Compared to the previously cited articles, this paper mainly presents two novel aspects. The first one is the inclusion of wind turbines within the modules of an MMC, whereas existing references have only studied the inclusion of solar panels and/or batteries. The second is the analysis of the power generation balancing between phases and arms; 
the literature had proposed regulators to perform such balancing, but this paper studies a case in which the system can be balanced automatically without the need to use regulators.

The article is structured as follows. Section 2 includes a short introduction to MMC and NLC. Section 3 explains the new topology and the control used. Section 4 provides a theoretical study of the variables involved in the power transfer between phases and between arms, which is necessary when the generation is unbalanced. Section 5 studies the case chosen to demonstrate that this configuration can work correctly even when there are large imbalances in the power generation. Finally, Section 6 presents the conclusions of the work.

\section{Fundamentals of Modular Multilevel Converters (MMC) and Near Level Control (NLC)}

\subsection{Fundamentals of $M M C$}

An introduction to the main elements that make up an MMC can be found in [21]; this sub-section includes the fundamental aspects of the MMC topology. The MMC features a three-phase topology, with two arms for each phase (Figure 1 top). Each arm consists of $\mathrm{n}$ SMs and an inductance $\mathrm{L}$ in series to smooth voltage differences. The most common $\mathrm{SM}$ topologies are $\mathrm{HB}$ and full-bridge (FB) (Figure 1 bottom). The operation of the HB is explained below, although that of the FB is similar; the main variables are included in Table 1. When the SM is in the ON state, the transistor T1 is in the ON state and the transistor T2 is in the OFF state; the SM voltage $v_{S M}$ is the same as that of the capacitor $v_{C}$; depending on whether the SM current $i_{S M}$ is positive or negative, the capacitor voltage increases or decreases, respectively. When the SM state is OFF, the transistor T2 is in the $\mathrm{ON}$ state and $\mathrm{T} 1$ is in the OFF state; the $\mathrm{SM}$ voltage $v_{S M}$ is zero, and the capacitor voltage $v_{C}$ remains constant.

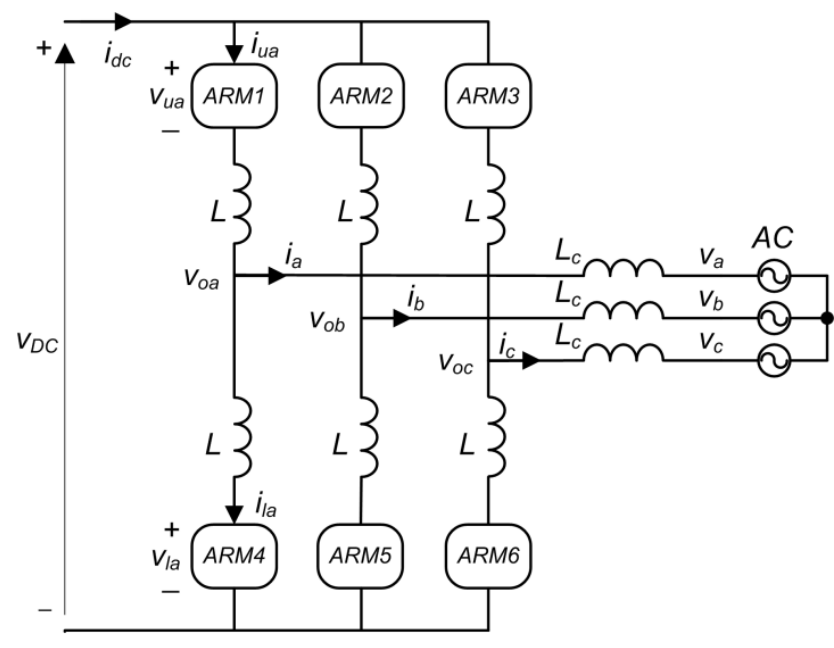

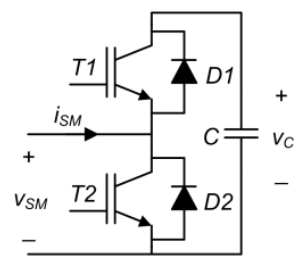

(a)

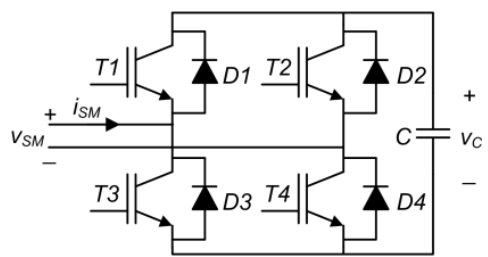

(b)

Figure 1. (Top) Topology of a modular multilevel converter (MMC) connected to the grid by means of an inductance. (Bottom) Switching modules (SM): (a) half-bridge (HB), and (b) full-bridge (FB) [21]. 
Table 1. Variables and states of a half-bridge (HB) switching module (SM) [21].

\begin{tabular}{ccccccc}
\hline SM State & $\mathbf{T}_{1}$ State & $\mathbf{T}_{2}$ State & $\boldsymbol{i}_{S M}$ & $\boldsymbol{\Delta} v_{C}$ & $\boldsymbol{i}_{S M}$ Flows through & $v_{S M}$ \\
\hline ON & ON & OFF & $>0$ & + & $\mathrm{D}_{1}$ & $v_{C}$ \\
ON & ON & OFF & $<0$ & - & $\mathrm{T}_{1}$ & $v_{C}$ \\
OFF & OFF & ON & $>0$ & 0 & $\mathrm{~T}_{2}$ & 0 \\
OFF & OFF & ON & $<0$ & 0 & $\mathrm{D}_{2}$ & 0 \\
\hline
\end{tabular}

The capacitor voltage is the ratio between the DC voltage $v_{D C}$ and the number of SMs of each arm $n, v_{C}=\frac{v_{D C}}{n}$. The sum of the number of modules in the ON state of the upper $n_{u}$ and lower $n_{l}$ arms is equal to $n$ :

$$
n_{u}+n_{l}=n
$$

The equation relating the voltage and current of the upper (lower) arm of the MMC is:

$$
v_{o a}= \pm \frac{V_{D C}}{2} \mp v_{u(l) a} \mp L \frac{d i_{u(l) a}}{d t}
$$

The voltage of the upper (lower) arm $v_{u(l) a}$ depend on the voltages of the capacitors of the SMs $v_{C u(l) a k}$ and the state of the SMs $S_{u(l) a k}$, which takes the value 1/0 depending on whether the state is ON/OFF.

$$
v_{u(l) a}=\sum_{k=1}^{n} S_{u(l) a k} v_{C u(l) a k}
$$

The upper (lower) arm current $i_{u(l) a}$ is the sum of half of the phase current $i_{a}$, one-third of the DC current $i_{d c}$ and the circulating current $i_{z a}$ [22]:

$$
i_{u(l) a}= \pm \frac{i_{a}}{2}+\frac{i_{d c}}{3}+i_{z a}
$$

From Equation (4) the circulating current is obtained:

$$
i_{z a}=\frac{i_{u a}+i_{l a}}{2}-\frac{i_{d c}}{3}
$$

The sum of the circulating currents of the three phases is zero:

$$
i_{z a}+i_{z b}+i_{z c}=0
$$

\subsection{Fundamentals of NLC}

Depending on whether the MMC has a low or high number of SM per arm, PWM or NLC is used, respectively. In the first case, a high switching frequency is required to ensure that the harmonics have a high frequency and can be eliminated using a filter connected between the converter and the mains. Due to the high switching frequency, the switching power losses are high. In the second case, the output voltage is built up by steps of the capacitor voltage of the SMs. The higher the number of SMs per arm, the higher the number of output voltage levels and the higher the number of eliminated low-frequency harmonics; therefore, the filtering requirements are reduced and the MMC can even be directly connected to the transformer. In this paper, an NLC has been used.

The switching period of the NLC can be fixed or variable. In the first case, the reference voltage is compared to the available levels with a constant period, as in Figure 2 top [23]. In the figure, it may seem that the period is not fixed because there are periods in which the NLC voltage does not change. In the second case (Figure 2 bottom), the change in the voltage level takes place when the reference crosses the intermediate value between two levels [24]. In this paper, a variable switching period is going to be used. 

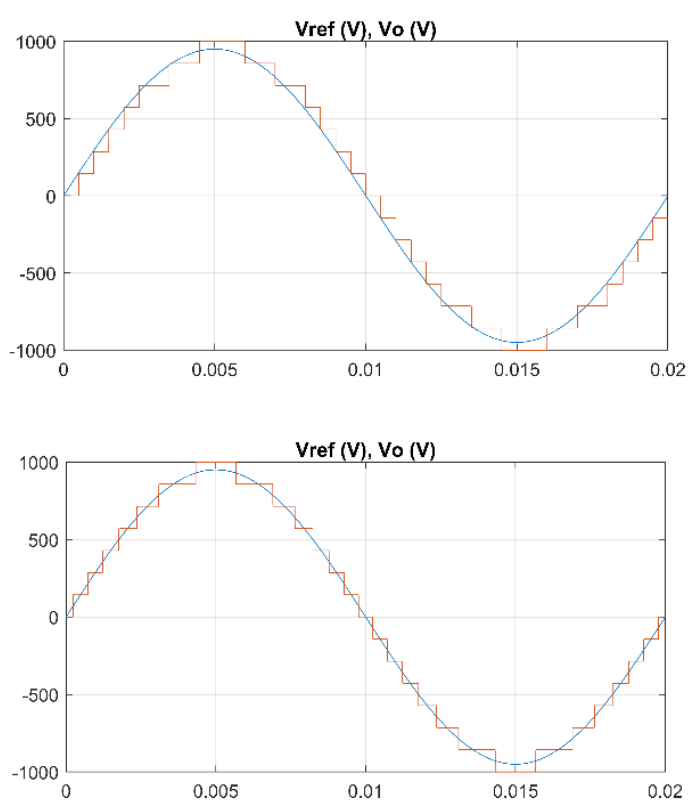

Figure 2. (Top) Reference voltage and near level control (NLC) voltage graphs when the switching period is fixed. (Bottom) Reference voltage and near level control (NLC) voltage graphs when the switching period is variable [21].

\section{Proposed Wind Farm Topology with NLC Modulation}

\subsection{Topology}

The classic topology of a wind farm with small or medium-sized wind turbines consists of units that are connected in parallel to the input of the grid connection transformer. Each of these units consists of a wind generator, two DC/AC converters, and a grid connection filter. The converter on the generator side is responsible for tracking the maximum power point (MPPT). The second converter is in charge of sending the generated power to the grid, using high-frequency modulation (PWM, SVM, etc.).

The proposed topology (Figure 3) is an MMC in which each SM has been replaced by a power module (PM) that includes the wind generator, the generator-side converter, and the HB of the SM. The generator-side converter handles the MPPT in the same way as in the classical configuration. This paper considers that the number of wind generators is high, so NLC modulation is used.

Each phase consists of two arms and two inductances. Each upper/lower arm includes $n_{u} / n_{l}$ PMs, which are connected in the ON/OFF state to make up the output voltage of each phase $v_{o i}$, so that the sum of the number of ON modules of the arms is equal to the number of PMs per arm $n, n_{u}+n_{l}=n$. The difference in the voltages of the upper and lower arms has a small value $v_{2 L}$, which causes the circulating currents. These currents can be obtained by dividing the voltage $v_{2 L}$ and the impedance of the two inductances $2 Z_{L}$.

This topology generates a multilevel grid connection voltage shaped by low-frequency steps. As the number of steps will be high, depending on the value of the transformer inductance, the converter can be connected to the transformer without using a filter. 

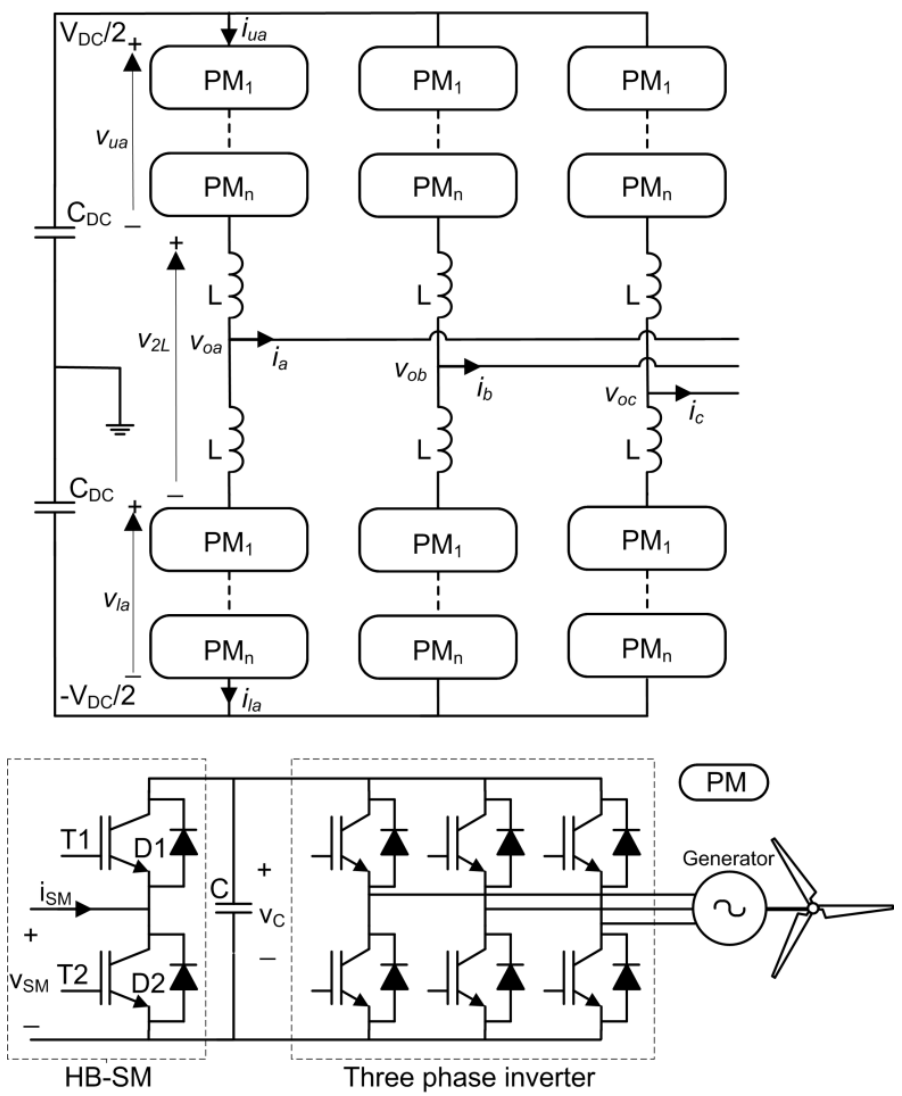

Figure 3. Proposed MMC for wind power generation, where the details of a power module (PM) including the wind generator, the generator-side converter, and the HB-SM can be seen.

This topology with this modulation has the following advantages over the classical configuration (summarized in Table 2):

(1). Fewer semiconductors. The classic configuration uses for each wind generator two three-phase DC / AC converters, i.e., 12 IGBT/MOSFETs, while the developed configuration uses one three-phase DC/ AC converter and one HB, in total 8 IGBT/MOSFETs.

(2). Lower switching losses. The classical configuration uses high-frequency modulation because it uses two-level converters; the configuration presented in this paper generates an output voltage with many phase-to-neutral voltage levels (e.g., 11 voltage levels for 10 PM per arm), so it is not necessary to use high-frequency modulation but it is more appropriate to use low-frequency modulation such as NLC. Thus, switching power losses are greatly reduced.

(3). Lower filtering requirements. The classic setup has a filter between the output converter and the grid to filter the two-level voltage of the converter; it uses one filter per generator. The proposed configuration generates the output voltage with a large number of steps so that the filtering requirements are very low or even eliminated, also, only one filter would be needed since there is only one converter connected to the grid. 
Table 2. Summary of the main differences between the two topologies.

\begin{tabular}{ccc}
\hline Number of Electric Generators $=\mathbf{N}_{\mathrm{EG}}$ & Classical Topology & Proposed Topology \\
\hline Electric generators & $\mathrm{N}_{\mathrm{EG}}$ & $\mathrm{N}_{\mathrm{EG}}$ \\
DC/AC converters & $2 \mathrm{~N}_{\mathrm{EG}}$ & $\mathrm{N}_{\mathrm{EG}}$ \\
Switching modules (SM) & 0 & $\mathrm{~N}_{\mathrm{EG}}$ \\
IGBTs & $12 \mathrm{~N}_{\mathrm{EG}}$ & $8 \mathrm{~N}_{\mathrm{EG}}$ \\
Switching frequency & High & Low \\
Switching losses & High & Low \\
Filters between converter and grid & $\mathrm{N}_{\mathrm{EG}}$ & $0 / 1$ \\
Wind turbine transformers & $0 / \mathrm{N}_{\mathrm{EG}}$ & 0 \\
Grid coupling transformer & $0 / 1$ & 1 \\
\hline
\end{tabular}

Additional aspects to compare are resilience, safety, and security. In the classical topology, when a DC / AC converter breaks down, its generator stops generating power, but the other generators remain supplying power as they are all connected in parallel. In the proposed topology, if the DC/AC converter on the generator side breaks down, the PM stops sending power but the MMC continues to operate. If the HB-SM fails, the entire MMC stops working as the PMs are connected in series. To avoid this, some additional semiconductors must be included in each PM to short-circuit the broken down PM. In terms of protection and safety, there are no major differences between the classic and the proposed topologies.

\subsection{Control Schemes}

The new converter can be controlled in the same way as a classic MMC with NLC modulation (Figure 4). The control consists of the external regulators (DC voltage and reactive power), the NLC modulator, and the PM capacitor balancing algorithm.

An external PI regulator is responsible for maintaining the DC voltage, $v_{D C}$. Another external regulator could be used for the converter reactive power, but it has been chosen to directly introduce a converter current reference on the $q$-axis, $i_{q}{ }^{*}$, of null value for simplicity; therefore, the converter will only deliver active power. The output of the DC voltage regulator is the converter current reference on the $d$-axis, $i_{d}{ }^{*}$. Then, two PI converters and the $d q$-axis decoupling equations [25] are responsible for generating the $d q$-axis converter voltage references, $v_{o d}{ }^{*}$ and $v_{o q}{ }^{*}$. It should be noted that the decoupling equations include the $\omega\left(L_{c}+L / 2\right)$ block, since the inductance appearing in series with the voltage source is the grid coupling inductance $L_{c}$ in series with the parallel of the two arm inductances $L$. Using the inverse Park and Clarke transformations, the references of the converter voltages in the three phases, $v_{o a}{ }^{*}, v_{o b}{ }^{*}$ and $v_{o c}{ }^{*}$, are obtained.

The NLC modulator in Figure 4 includes a modulator for each of the three phases. Its input is the reference voltage of the corresponding phase. It takes samples of its input voltage, with a period $T_{\text {reg, }}$, and chooses the voltage level closest to the voltage reference. It then translates that voltage level into the number of PMs that must be in the ON state, on the upper arm $n_{u i}$ and on the lower arm $n_{l i}$, to achieve that level of the converter output voltage. The output voltage of the converter $v_{o i}$ can take the values $-\frac{V_{D C}}{2}+\frac{V_{D C}}{n} n_{l i}$. To obtain the desired value of the output voltage, a number of modules $n_{l i}$ in the ON state is chosen in the lower arm and the number of modules in the ON state in the upper arm is obtained using Equation (1).

Finally, to balance the voltages of the PM capacitors, a conventional algorithm $[10,26]$ is used, which sorts the voltages of the PM capacitors of the same arm, upper $v_{c u i j}$ or lower $v_{c l i j}$ (with $j=1$ to $n$ ), and chooses which ones to turn ON depending on the direction of the arm current, upper $i_{u i}$ or lower $i_{l i}$. This algorithm is run for each of the six arms. Its inputs are the number $n_{u(l) i}$ of PMs in the ON state of the upper (lower) arm and the direction of the arm current. If the current is positive, the algorithm chooses the $n_{u(l) i} \mathrm{PMs}$ with the lowest voltage, so that the positive current is responsible for charging it. If the 
current is negative, the algorithm chooses the most charged $n_{u(l) i}$ capacitors, so that the negative current discharges them.

To obtain the angle $\theta$ and the angular speed $\omega$ of the grid voltage vector, a dq-PLL is used [27].
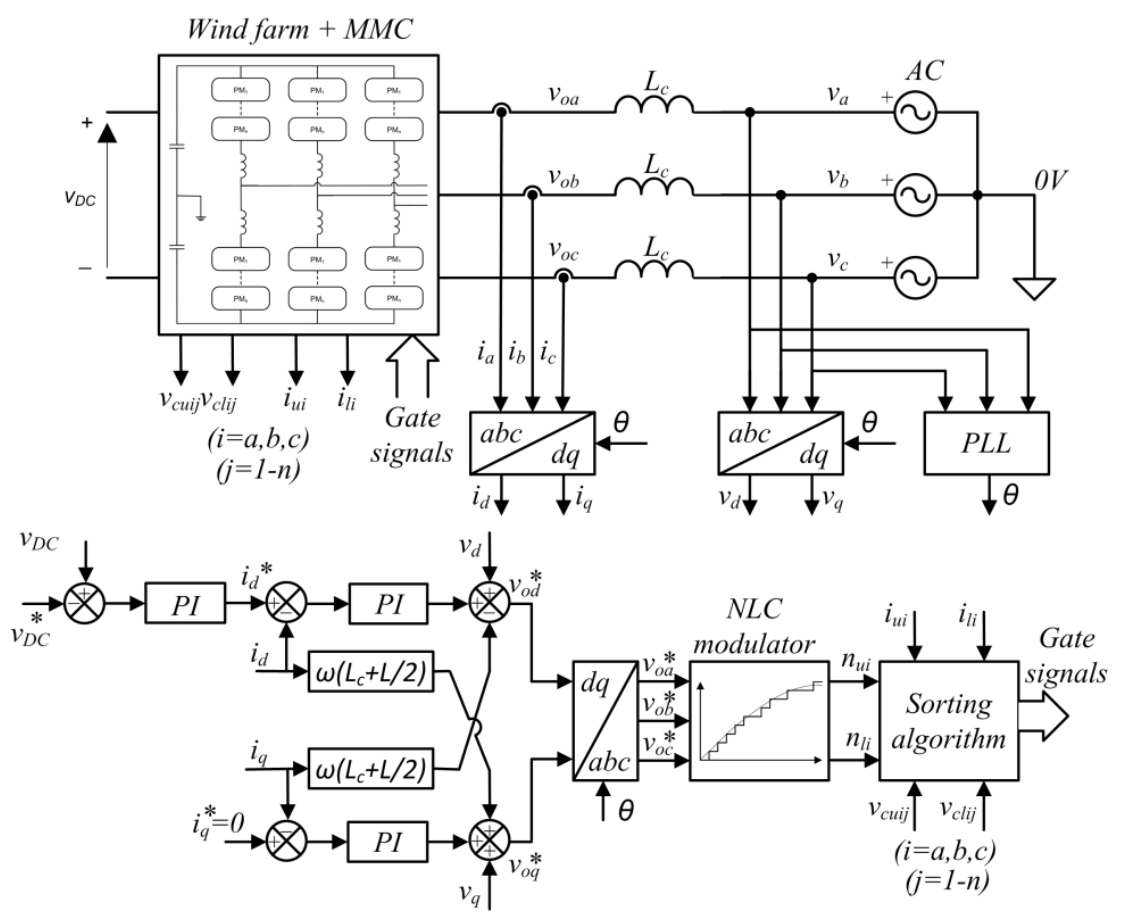

Figure 4. Control scheme of the new topology that integrates the wind generators into the structure of an MMC and uses an NLC modulator.

\section{Power Transfer between Phases and between Arms}

This section addresses the situation that takes place when there are generation imbalances between phases and between arms. It must be taken into account that the vector control system proposed in Figure 4 delivers the same power to the grid through each of the three phases. Therefore, if each phase generates a different power, there must be a mechanism that allows power transfer between the phases. Regarding the arms, the converter tends to deliver half of the phase power from each of the arms, because half of the current of each phase flows through each of the arms that are connected to it. Therefore, there must be a mechanism to transfer power between the two arms of a phase, or, in other words, to send different powers from each of the two arms.

Generation imbalances within the PMs of the same arm do not cause any problem, since they are balanced by the PM capacitor voltage ordering algorithm.

The best situation is when there is balance in the generation of all wind generators, but there will be cases where the generation is unbalanced. Therefore, the four possible cases are listed in Table 3.

Table 3. Cases that can be found regarding the balance or imbalance between arms and phases.

\begin{tabular}{cl}
\hline $\mathbf{N}^{\circ}$ & \multicolumn{1}{c}{ Cases } \\
\hline 1 & $\begin{array}{l}\text { Balanced generation: all wind generators generate the same power. } \\
\text { Imbalance between phases: generators of the same phase generate the same power, } \\
\text { which is different from that generated by turbines of a different phase. }\end{array}$ \\
3 & $\begin{array}{l}\text { Imbalance between arms: all phases generate the same total power, but their upper and } \\
\text { lower arms generate different power. } \\
\text { Imbalance between phases and arms. It is a mixture of cases } 2 \text { and 3, so it will not } \\
\text { be studied }\end{array}$ \\
\end{tabular}


The vector control ensures that each phase delivers the same AC power so that the system is balanced. If the power generated in each phase and/or in each arm is different, there must be internal power balancing mechanisms, otherwise, the system would be unbalanced. This analysis is made in detail below.

\subsection{Balanced Generation}

In this case, all wind turbines generate the same power, so the power generated in the arms and the phases is balanced. No power has to be transferred between phases or arms so that the AC power delivered through each phase is the same.

\subsection{Imbalance between Phases}

In this case, it will be considered that the power generated by the wind turbines of each phase is the same, but that the power generated by each turbine of one phase is different from the power generated by the turbine of another phase. Since the AC power to be delivered in each phase must be the same, there must be power transfer from the phases that generate more power to the phases that generate less power.

The continuous component of the circulating current of phase $i, i_{Z D C}$, is responsible for sending/receiving power on phase $i$ to/from other phases. The circulating current of phase $i, i_{Z i}$, consists of a continuous component $i_{Z D C i}$, the first harmonic of the alternating component $(50 \mathrm{~Hz}) i_{\mathrm{ZACl} i}$ and the second harmonic of the alternating component $(100 \mathrm{~Hz}) i_{\mathrm{ZAC} 2 i}$,

$$
i_{Z i}=i_{Z D C i}+i_{Z A C 1 i}+i_{Z A C 2 i}
$$

The product of the DC component of the circulating current $i_{Z D C i}$ by the DC voltage $v_{D C}$ is the power absorbed by that phase, and which has been sent from the other phases. By reversing the sign of this power, the power delivered by phase $i, p_{Z i}$, to the other two phases is obtained.

$$
p_{Z i}=-i_{Z D C i} \cdot v_{D C}
$$

Since the sum of the circulating currents of the three phases is zero, the sum of the continuous components of the three circulating currents and the sum of the powers transferred between the three phases will also be zero.

$$
\begin{gathered}
i_{Z D C a}+i_{Z D C b}+i_{Z D C c}=0 \\
p_{Z a}+p_{Z b}+p_{Z c}=0
\end{gathered}
$$

In the analysis of the sub-cases of Sections 5.2.1 and 5.2.2, it will be seen that this power transfer occurs naturally without the use of any type of regulator.

\subsection{Imbalance between Arms}

In this case, it is going to be considered that the power generated by the three upper arms of the three phases is equal $\left(p_{\text {WTua }}=p_{W T u b}=p_{W T u c}=p_{W T u}\right)$, that the power generated by the three lower arms of the three phases is equal $\left(p_{\text {WTla }}=p_{W T l b}=p_{W T l c}=p_{W T l}\right)$, but that the power generated by the upper and lower arms is different, $p_{W T u} \neq p_{W T l}$. In this situation, the power generated by each phase is the same and it is the sum of the powers of the upper and lower arms, $p_{W T a}=p_{W T b}=p_{W T c}=p_{W T u}+p_{W T l}$. Therefore, there is no need to transfer power between the phases.

In the absence of a $50 \mathrm{~Hz}$ circulating current, the power supplied by the upper and lower arms is the same, because the current of each phase of the converter is divided by $50 \%$ between the two arms, upper and lower. Since the AC voltage of the upper and lower arms is equal, and the arm power is the product of the $50 \mathrm{~Hz}$ voltage and the $50 \mathrm{~Hz}$ current, then the powers of the upper and lower arms would be equal. For the upper and lower arms to be able to deliver an AC power equal to the power they generate (different from each other), there must be a $50 \mathrm{~Hz}$ circulating current that, according to Equation (4) and 
considering that $i_{d c}=0$, allows it. In the following, all this will be analyzed in detail and the equations that describe it will be obtained.

From Equation (4), and taking into account that $i_{d c}=0$, the current of the upper (lower) arm is:

$$
i_{u(l) i}= \pm \frac{i_{i}}{2}+i_{Z i}
$$

The power delivered by the upper (lower) arm of phase $i$ in $50 \mathrm{~Hz} \mathrm{AC}$ is:

$$
p_{u(l) i}=-v_{u(l) A C 1 i} \cdot i_{u(l) A C 1 i}
$$

where the subscript " 1 " means the first harmonic $(50 \mathrm{~Hz})$. The $50 \mathrm{~Hz}$ voltage of the upper (lower) arm $v_{u(l) A C 1 i}$ has a value approximately equal to the converter output voltage $v_{o i}$, assuming that the voltage on the arm inductance is very small,

$$
v_{u(l) A C 1 i}=\mp v_{o i}
$$

The $50 \mathrm{~Hz}$ circulating current $i_{Z A C 1 i}$ is the quotient of the voltage of the two arm inductances $v_{2 L A C 1 i}$ and the impedances of the two arm inductances $2 Z_{L}$,

$$
i_{Z A C 1 i}=\frac{v_{2 L A C 1 i}}{2 Z_{L}}
$$

where the voltage of the two inductances $v_{2 L A C 1 i}$ is obtained as

$$
v_{u A C 1 i}+v_{2 L A C 1 i}+v_{l A C 1 i}=0 \rightarrow v_{2 L A C 1 i}=-\left(v_{u A C 1 i}+v_{l A C 1 i}\right)
$$

From Equations (11)-(13), the expression of the power generated in the upper (lower) arm is obtained,

$$
p_{u(l) i}= \pm v_{o i} \cdot\left( \pm \frac{i_{i}}{2}+i_{Z A C 1 i}\right)
$$

Given Equation (16), it is observed that the circulating current $i_{Z A C 1 i}$ allows that the power delivered by the upper and lower arms can be different, which is what is desired when the generation is different. This circulating current is generated due to the difference between the voltages of the two arms, $v_{u A C 1 i}$ and $v_{l A C 1}$, which occurs due to the voltage differences of the upper and lower arm capacitors.

The voltage of the first $\mathrm{AC}$ harmonic of the upper $v_{u A C 1 i}$ and lower $v_{l A C 1 i}$ arms is generated by NLC modulation, the levels of which are formed by the voltage of the capacitors of the SMs. If the voltage of these capacitors increases or decreases a little, the peak value of the voltages $v_{u A C 1 i}$ and $v_{l A C 1 i}$ also increases or decreases a little. This is what happens when there is an imbalance in the generation between the upper and lower arms. If, for example, the generation of the upper arm is lower than that of the lower arm, the voltage of the capacitors of the upper and lower arms is reduced and increased a little, respectively, until a circulating current is generated, according to Equation (14), which allows the power of the wind generators of the arm to be equal to the power delivered by that arm to the output of the converter. All this can be seen by the examples in Sections 5.3.1 and 5.3.2 and is explained graphically below in Figure 5 . In that, the arm voltage vectors $v_{u(l) A C 1 i}$ have been placed (for simplicity the subscripts $u(l) A C 1 i$ are replaced by $u(l)$ ), from which the value of $v_{2 L A C 1}$, called in a simplified form $v_{2 L}$, is obtained by Equation (15). By means of Equation (14), $i_{Z A C 1 i}$, called $i_{Z}$, is obtained; and by means of Equation (11), the currents of the arms, $i_{u(l)}$, are obtained. The active power delivered by each arm is calculated by means of the rms values of the voltages and currents:

$$
P_{u(l)}=v_{u(l) r m s} i_{u(l) r m s} \cos \varphi_{u(l)}=v_{u(l) r m s} i_{u(l) x r m s}
$$


As can be seen in Figure 5, the presence of the circulating current allows the currents $i_{u(l) x r m s}$ to have different values and therefore the powers $P_{u(l)}$ to have different values in turn.

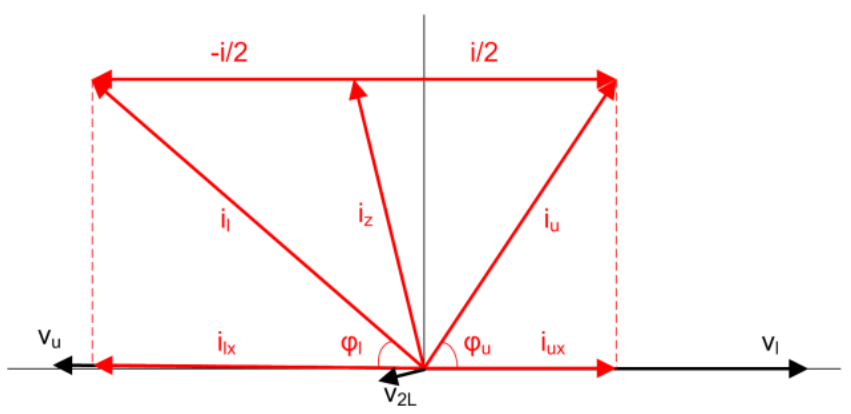

Figure 5. Vector diagram of the variables involved in power transmission from the arms. All of them are $50 \mathrm{~Hz}$ variables (harmonic 1).

\subsection{Imbalance between Phases and Arms}

This case is not analyzed because it is a combination of the cases of Sections 4.2 and 4.3, already analyzed.

\section{Analysis of a Case for Different Generation Scenarios}

In this section, a case (a topology and control) to integrate a large number of wind generators into the structure of an MMC has been proposed. Specific parameters of capacitors and inductances have been selected, together with the parameters of the PI regulators, by trial and error to obtain a good performance for very wide operating ranges. The following sub-cases have been tested: balanced generation of all generators for high and medium powers, unbalanced generation between phases when these imbalances are medium and when they are large, and unbalanced generation between arms for medium and large imbalances. The analysis has been carried out by simulation with Matlab/Simulink, using the parameters shown in Table 4. These parameters have been chosen by trial and error to obtain a balance between several parameters: $100 \mathrm{~Hz}$ circulating current amplitude, capacitor voltage ripple, DC voltage ripple, and dynamic response. A model replaces the wind generator with a current source of a value equal to the quotient of the wind generator power $P_{W T}$ and the PM capacitor voltage $v_{C}$ (Figure 6). The study method presented in this paper, as indicated above, is based on the interpretation of the data obtained by means of the simulation in Matlab/Simulink of the models shown in Figures 3 and 4. In order to find an optimal relationship between the simulation time and the reduction of errors in the data obtained in the simulation, it was chosen:

- Discrete simulation.

- Fixed-step. The sample time was set at $T_{S}=10 \mu \mathrm{s}$.

- Solver: Bogacki-Shampine.

Table 4. Simulation parameters of the case under analysis.

\begin{tabular}{cccc}
\hline Parameter & Value & Parameter & Value \\
\hline$n$ & 10 & $L_{c}$ & $15 \mathrm{mH}$ \\
$T_{S}$ & $10 \mu \mathrm{s}$ & $i_{q}{ }^{*}$ & 0 \\
$T_{r e g}$ & $130 \mu \mathrm{s}$ & $k_{p, V D C}$ & 0.64 \\
$v_{D C}$ & $2.4 \mathrm{kV}$ & $k_{i, V D C}$ & 1.6 \\
$v_{p h, p h}$ & $1300 V_{R M S}$ & $k_{p, i d, i q}$ & 2.5 \\
$C$ & $120 \mathrm{mF}$ & $k_{i, i d, i q}$ & 25 \\
$C_{D C}$ & $40 \mu \mathrm{F}$ & $k_{p, P L L}$ & 0.2 \\
$L$ & $375 \mu \mathrm{H}$ & $k_{i, P L L}$ & 2 \\
\hline
\end{tabular}




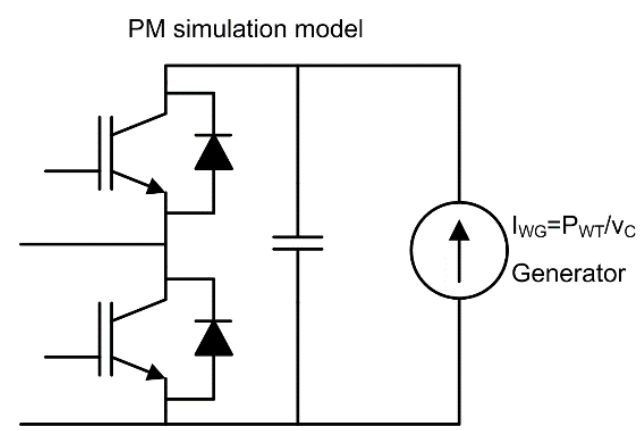

Figure 6. PM simulation model, where the wind generator has been replaced by a current source.

Three cases have been chosen: balanced generation, phase imbalance, and arm imbalance. Only the case of simultaneous phase and arm unbalance is missing, but it is a combination of the last two cases. Balanced generation cases correspond to actual cases where all generators are running and wind speeds are average or high. Phase or arm differences correspond to actual cases where generation is different or even zero for some generators. Generation differences may be transient due to gusty winds or permanent due to the location of the turbines. Null generation is due to broken generators or generators under maintenance.

The events that one or more turbines may suffer are, mainly, the reduction of power caused by the decrease in wind speed, and the non-generation of power when the turbine is out of service or undergoing maintenance. Both cases are included within the cases studied, because in both the power generated by the arm and/or the phase is reduced.

The imbalances to be analyzed are very large, so much so that they will probably not occur in actual applications, but this will ensure the correct operation of the system. In addition, when inter-arm imbalances occur, the case where several generators stop working will be simulated, which is a tougher case than if the output of all generators is reduced by the same amount.

\subsection{Balanced Generation}

\subsubsection{Balanced Generation and Medium Power}

In this subsection and the following one, two situations are studied in which all the PMs generate the same power; in this subsection, they generate half the rated power, $750 \mathrm{~W}$, and in the following subsection, they generate the rated power, $1500 \mathrm{~W}$.

Since all generators deliver the same power of $750 \mathrm{~W}$, each phase delivers a power $p_{a}=p_{b}=p_{c}=15 \mathrm{~kW}$. The simulation results are presented in Figure 7. It is observed that the output voltage of the MMC $v_{\text {oabc }}$ (Figure 7a) has 11 levels, as corresponds to $10 \mathrm{SM}$ per arm and submits a correct waveform; the output currents of the MMC $i_{a b c}$ (Figure $7 \mathrm{~b}$ ) also have a correct waveform. The output voltage $v_{\text {oabc }}$ waveform (Figure $7 \mathrm{a}$ ) is the same in all cases, so it will not be repeated in each case. The power transferred between phases $p_{z a b c}$ (Figure 7c) is zero (only a high frequency ripple) because there is no imbalance between the phases. The power delivered by each phase $p_{a b c}$ (Figure $7 \mathrm{~d}$ ) is equal to $15 \mathrm{~kW}$ in each of the three phases, the same as the power generated by each phase. The voltage on the DC link $v_{D C}$ (Figure 7e) has a small peak during start-up and then remains constant with a very small ripple. The circulating currents $i_{z a b c}$ (Figure 7f) have a moderate value (Table 5) of $3.64 \mathrm{~A}$ for the $100 \mathrm{~Hz}$ harmonic and $2.08 \mathrm{~A}$ for the $50 \mathrm{~Hz}$ harmonic. The last two plots (Figure $7 \mathrm{~g}$ ) show the values of the capacitor voltages of the upper $v_{c u a(1-10)}$ and lower $v_{c l a(1-10)}$ arm modules, which are overlapped. A small ripple of about $0.5 \mathrm{~V}$ and average voltages of about $240 \mathrm{~V}$ are observed, both in the capacitors of the upper and lower arms. 


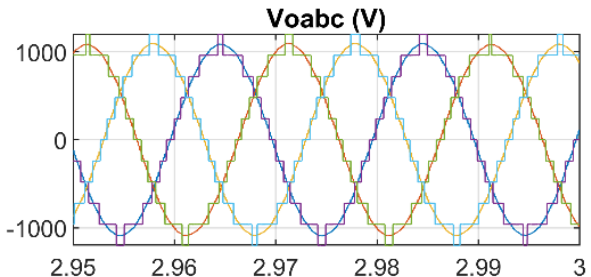

(a)

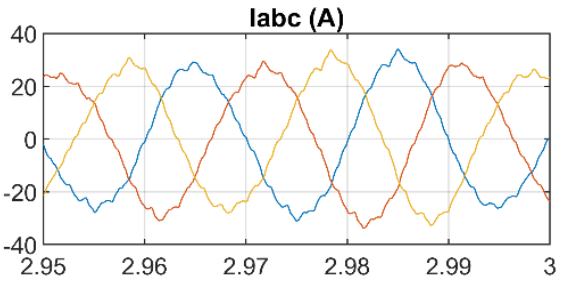

(b)

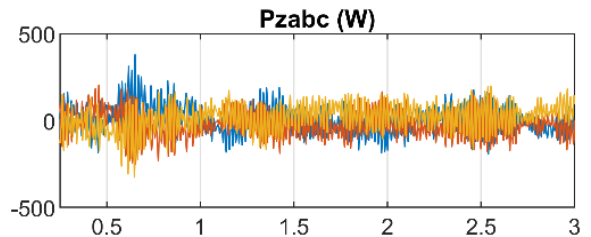

(c)

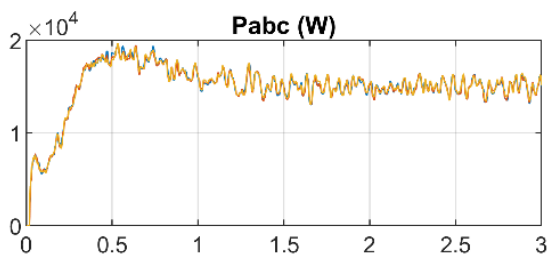

(d)

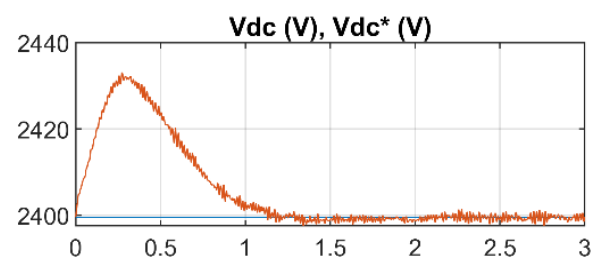

(e)

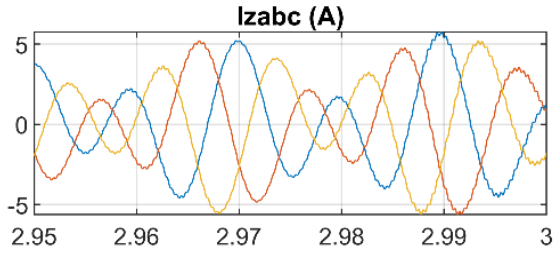

(f)

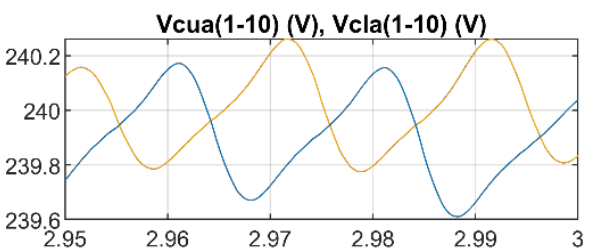

(g)

Figure 7. Simulation graphs when the power is medium and the generation is balanced. (a) Output voltages. (b) Output currents. (c) Power transferred between phases. (d) Power delivered by each phase. (e) Voltage on the DC link. (f) Circulating currents. (g) Capacitor voltages.

Table 5. Summary of the peak values of the variables obtained in the simulation. Voltages are in volts, currents in amperes, and angles in degrees. All variables are $50 \mathrm{~Hz}$ except where noted.

\begin{tabular}{|c|c|c|c|c|c|c|c|}
\hline $\begin{array}{l}\text { Sub- } \\
\text { Section }\end{array}$ & $i_{a}$ & $i_{u a}$ & $i_{l a}$ & $v_{u a}$ & $v_{l a}$ & $i_{z a}$ & $\begin{array}{r}i_{z A C 2 a} \\
(100 \mathrm{HZ})\end{array}$ \\
\hline 5.1 .1 & $28.47 \mathrm{~L} 0.0^{\circ}$ & $14.42 \mathrm{~L}-8.3^{\circ}$ & $14.35 \mathrm{~L} 188.3^{\circ}$ & $1069.99 \mathrm{~L} 187.2^{\circ}$ & $1069.23 \mathrm{~L} 7.2^{\circ}$ & $2.08 \mathrm{~L}-89.0^{\circ}$ & $3.64 \mathrm{~L} 98.3^{\circ}$ \\
\hline 5.1 .2 & $56.87 \mathrm{~L}-0.7^{\circ}$ & $28.65 \mathrm{~L}-1.9^{\circ}$ & $28.23 \mathrm{~L} 180.6^{\circ}$ & 1096.65L194.2 & 1096.37L14.2 ${ }^{\circ}$ & $0.64 \mathrm{~L}-71.8^{\circ}$ & $7.11 \mathrm{~L} 107.0^{\circ}$ \\
\hline 5.2 .1 & $27.08 \mathrm{~L} 1.2^{\circ}$ & $12.08 \mathrm{~L}-4.5^{\circ}$ & $15.10 \mathrm{~L} 185.8^{\circ}$ & $1062.72 \mathrm{~L} 186.7^{\circ}$ & 1062.30L6.7 ${ }^{\circ}$ & $1.94 \mathrm{~L} 219.7^{\circ}$ & $3.16 \mathrm{~L} 95.0^{\circ}$ \\
\hline 5.2 .2 & $28.86 \mathrm{~L}-1.6^{\circ}$ & $13.25 \mathrm{~L}-5.2^{\circ}$ & $15.65 \mathrm{~L} 181.4^{\circ}$ & $1072.55 \mathrm{~L} 187.1^{\circ}$ & $1072.32 \mathrm{~L} 7.1^{\circ}$ & $1.45 \mathrm{~L} 212.8^{\circ}$ & 3.13L101.7 ${ }^{\circ}$ \\
\hline 5.3 .1 & $46.16 \mathrm{~L}-1.0^{\circ}$ & 29.37L67. $1^{\circ}$ & $44.53 \mathrm{~L} 141.3^{\circ}$ & 1082.10L191.5 & $1089.03 \mathrm{~L} 11.6^{\circ}$ & 29.84L113.0 & $5.58 \mathrm{~L} 104.0^{\circ}$ \\
\hline 5.3 .2 & $32.61 \mathrm{~L} 1.1^{\circ}$ & $46.63 \mathrm{~L} 93.6^{\circ}$ & $58.08 \mathrm{~L} 127.7^{\circ}$ & 1064.22L188.0 & $1075.34 \mathrm{~L} 8.2^{\circ}$ & $50.08 \mathrm{~L} 112.6^{\circ}$ & $4.27 \mathrm{~L} 98.0^{\circ}$ \\
\hline
\end{tabular}

\subsubsection{Balanced Generation and Full Power}

In this case, all generators deliver the same power of $1.5 \mathrm{~kW}$, which is their maximum or rated power, and therefore each phase delivers $p_{a}=p_{b}=p_{c}=30 \mathrm{~kW}$. As can be seen in Figure 8a, the MMC output currents $i_{a b c}$ are correct. The DC power transmitted between phases $p_{z a b c}$ (Figure $8 \mathrm{~b}$ ) is zero, except for a small ripple, because all phases produce the same power. The power sent to the grid $p_{a b c}$ (Figure $8 \mathrm{c}$ ) is equal in all three phases and equal to the power generated in each phase, $30 \mathrm{~kW}$. The voltage on the DC link $v_{D C}$ (Figure $8 \mathrm{~d}$ ) peaks at $60 \mathrm{~V}$ during start-up and then remains constant. The circulating current $i_{z a b c}$ has admissible values, $7.11 \mathrm{~A}(100 \mathrm{~Hz})$ and $0.64 \mathrm{~A}(50 \mathrm{~Hz})$ (Table 5). The 
capacitor voltages of the upper $v_{c u a(1-10)}$ and lower $v_{c l a(1-10)}$ modules (Figure 8e) have a ripple of about $1 \mathrm{~V}$ and an average value of about $240 \mathrm{~V}$, which are appropriate values.

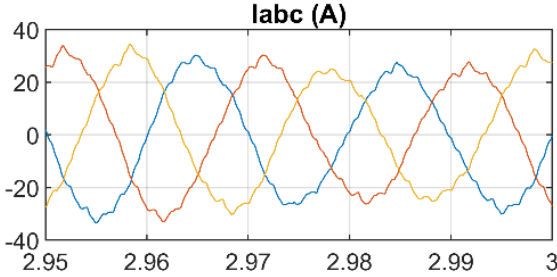

(a)

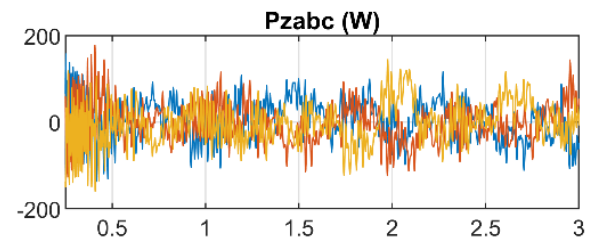

(b)

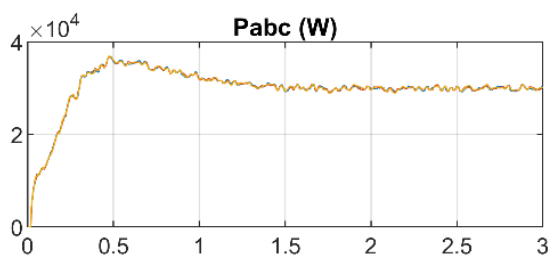

(c)

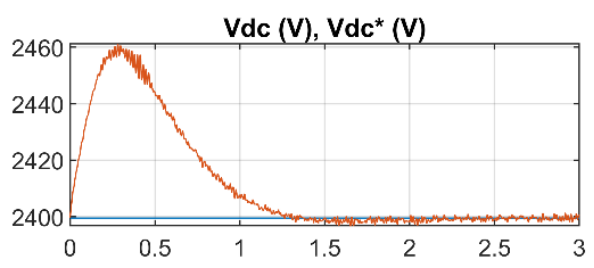

(d)

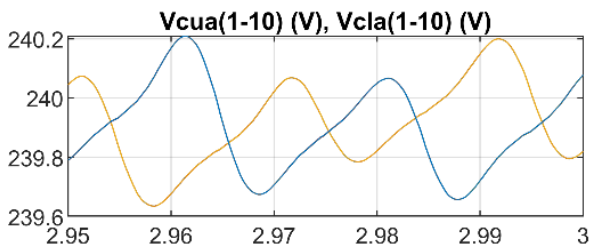

(e)

Figure 8. Simulation graphics when the generation is balanced and the power is full. (a) Output currents. (b) Power transferred between phases. (c) Power delivered by each phase. (d) Voltage on the DC link. (e) Capacitor voltages.

\subsection{Imbalance between Phases}

\subsubsection{Medium Imbalance between Phases}

In this sub-section and the following one, the situation of generation imbalance between phases is addressed. In this sub-section, a medium imbalance is simulated and in the next one, a large imbalance is simulated.

Each of the 20 generators in arms a, b, and c supply $500 \mathrm{~W}, 750 \mathrm{~W}$, and $1000 \mathrm{~W}$ each, respectively. The total power generated by each phase is: $p_{W T a}=10 \mathrm{~kW}, p_{W T b}=15 \mathrm{~kW}$, $p_{W T c}=20 \mathrm{~kW}$. Therefore, there is a transfer of $5 \mathrm{~kW}$ of power from the third phase $\left(p_{z c}\right)$ to the first phase $\left(p_{z a}\right)$, as can be seen in Figure $9 \mathrm{~b}$. Once this transfer of power between phases is carried out, the power delivered by each phase in AC $p_{a b c}$ is equal in the three phases, and having a value equal to the average power, $15 \mathrm{~kW}$ (Figure 9c). It is observed in Figure $9 \mathrm{a}$ that the MMC currents $i_{a b c}$ are correct. The DC link voltage $v_{D C}$ has a small peak of $30 \mathrm{~V}$ during start-up and then remains constant (Figure 9d). The circulating currents of the first $i_{z a}$ and third $i_{z c}$ phases have a DC value due to the power transfer between these phases; in addition, they have small AC values (Table 5), $3.16 \mathrm{~A} \mathrm{(100} \mathrm{Hz)} \mathrm{and} 1.94 \mathrm{~A}$ $(50 \mathrm{~Hz})$. The voltage ripple of the module capacitors is small (Figure 9e), about $0.5 \mathrm{~V}$, and their average value is $240 \mathrm{~V}$, both for those of the upper arms $v_{c u a(1-10)}$ and those of the lower ones $v_{\text {cla }}(1-10)$.

As indicated in Section 4.2, the generation differences between phases are balanced naturally, without the need for a regulator, by the continuous component of the circulating currents. The power received by phase a from the other phases is the product of the DC voltage $(2400 \mathrm{~V})$ by the average value of the circulating current of phase a $(1.96 \mathrm{~A})$, whose product is $4584 \mathrm{~W}$. The power delivered by phase $\mathrm{c}$ to the other phases is the product of the DC voltage $(2400 \mathrm{~V})$ by the average value of the circulating current of phase c (1.91 A), 
whose product is $4992 \mathrm{~W}$. These values match the powers generated in each of the phases $\left(p_{W T a}=10 \mathrm{~kW}, p_{W T b}=15 \mathrm{~kW}, p_{W T c}=20 \mathrm{~kW}\right)$ so that the power delivered to the grid is balanced.

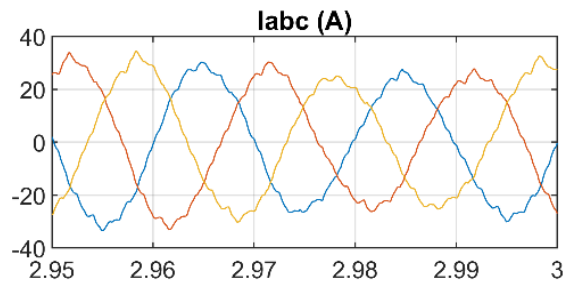

(a)

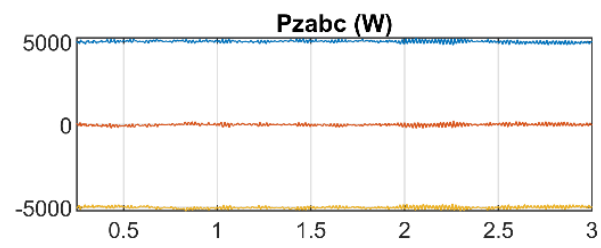

(b)

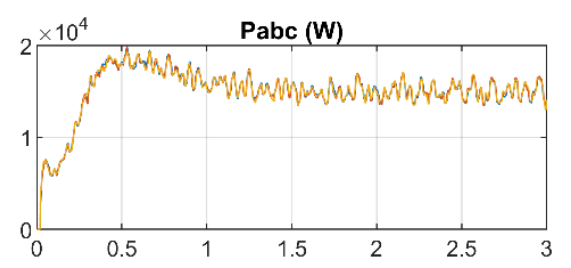

(c)

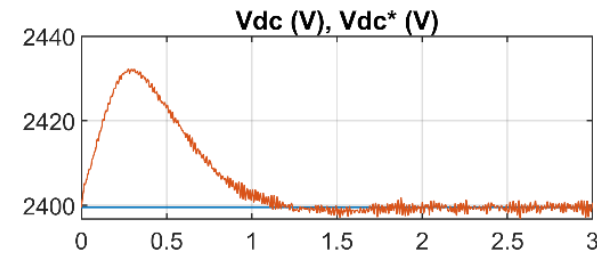

(d)

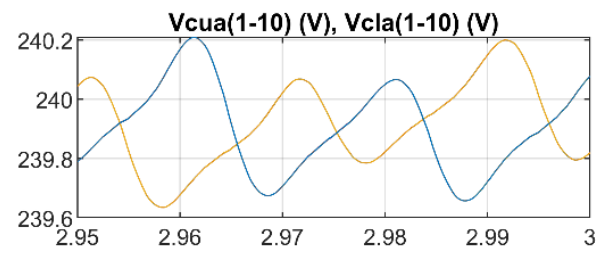

(e)

Figure 9. Simulation graphs when the generation has a medium phase imbalance. (a) Output currents. (b) Power transferred between phases. (c) Power delivered by each phase. (d) Voltage on the DC link. (e) Capacitor voltages.

\subsubsection{Large Imbalance between Phases}

In this case, there is a large imbalance between the generation of the different phases; the generators of arms a, b, and c supply $0,750 \mathrm{~W}$, and $1500 \mathrm{~W}$ each, respectively. The total power supplied by each phase is: $p_{W T a}=0 \mathrm{~kW}, p_{W T b}=15 \mathrm{~kW}, p_{W T c}=30 \mathrm{~kW}$. Figure 10 shows that all variables are correct. The currents $i_{a b c}$ of the MMC (Figure 10a) have appropriate values. The values of the transferred powers between phases $p_{z a b c}$ (Figure 10b) indicate that phase $\mathrm{c}$ transfers a power of $15 \mathrm{~kW}$ towards phase a. Thus, each of the three phases transfers the same AC power to the grid, $p_{a b c}=15 \mathrm{~kW}$ (Figure 10c). The DC voltage $v_{D C}$ (Figure 10d) is constant after a small start-up peak. The circulating currents $i_{z a}$ and $i_{z c}$ have DC values because they transfer power between phases a and c; the AC components have moderate values (Table 5), $3.13 \mathrm{~A}(100 \mathrm{~Hz})$ and $1.45 \mathrm{~A}(50 \mathrm{~Hz})$. The capacitors of the modules have a ripple of $0.4 \mathrm{~V}$ and an average value of $240 \mathrm{~V}$ (Figure 10e), with no difference between the capacitors of the upper and lower arms.

As in Section 5.2.1, and as indicated theoretically in Section 4.2, the generation differences between the phases are balanced naturally, without the need for a regulator, by the DC component of the circulating currents. The power received by phase a from the other phases is the product of the DC voltage $(2400 \mathrm{~V})$ by the average value of the circulating current of phase a $(6.12 \mathrm{~A}), 14,688 \mathrm{~W}$. The power delivered by phase $\mathrm{c}$ to the other phases is the product of the DC voltage $(2400 \mathrm{~V})$ by the average value of the circulating current of phase c (6.21 A), 14,904 W. 


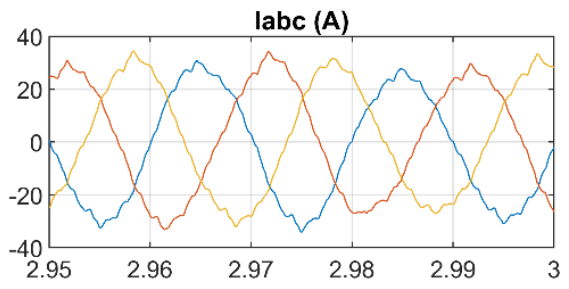

(a)

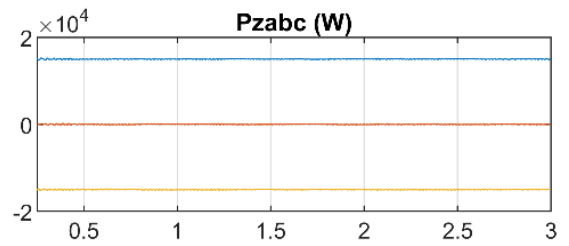

(b)

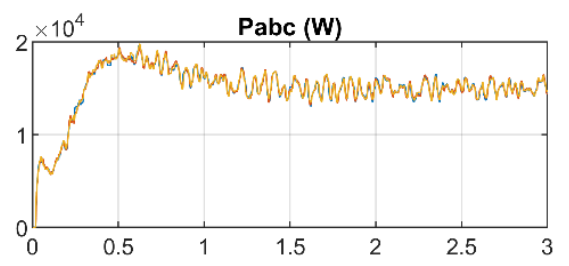

(c)

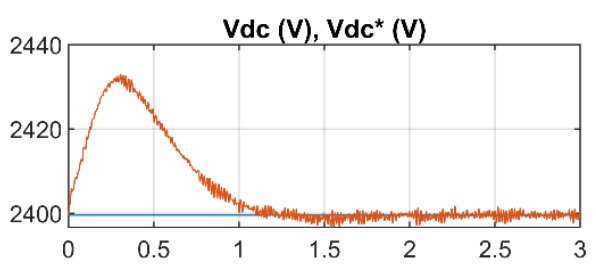

(d)

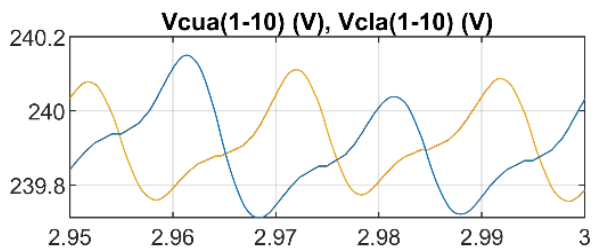

(e)

Figure 10. Simulation graphs when the generation has a large imbalance between phases. (a) Output currents. (b) Power transferred between phases. (c) Power delivered by each phase. (d) Voltage on the DC link. (e) Capacitor voltages.

\subsection{Imbalance between Arms}

\subsubsection{Medium Imbalance between Arms}

In this sub-section and the following one, the three phases generate the same power but there is an imbalance between the upper and lower arms. This sub-section analyzes a medium imbalance situation and the following one a very high imbalance situation.

The power generated by each phase is the same, $p_{W T a}=p_{W T b}=p_{W T c}=24 \mathrm{kWW}$, but the power generated in the upper and lower arms of each phase has a medium imbalance, $p_{u i}=9 \mathrm{~kW}$ and $p_{l i}=15 \mathrm{~kW}$. For this, all generators supply $1500 \mathrm{~W}$ except 4 generators in each upper arm which generate zero power. As can be seen in the graph of the powers of the upper arm $p_{u a}$ and lower arm $p_{l a}$ of the first phase (see Figure 11b), the upper arm generates $9 \mathrm{~kW}$ and the lower arm $15 \mathrm{~kW}$. Despite this, an equilibrium situation has been reached where it is observed that each phase delivers the same power to the grid $p_{a}=p_{b}=p_{c}=24 \mathrm{~kW}$ (Figure 11c). This is due to the presence of a $50 \mathrm{~Hz}$ circulating current of $29.84 \mathrm{~A}$ (see Table 5), much higher than in the previous sub-cases (without imbalance between arms). This current is responsible for getting each arm to deliver the same power it generates, as explained theoretically in Section 4.3. The $100 \mathrm{~Hz}$ component of the circulating current remains at similar values to the previous sub-cases, 5.58 A (Table 5).

All other variables remain at similar values to the previous cases. The MMC currents have appropriate values (Figure 11a). The DC voltage has a small start-up peak and a small steady-state ripple (Figure 11d). The capacitor voltages (Figure 11e) have ripples slightly higher than $1 \mathrm{~V}$ and a small difference, of about $1 \mathrm{~V}$, between the average voltage of the upper and lower capacitors.

As explained in Section 4.3, the small differences between the average value of the upper and lower capacitor voltages (approximately $1 \mathrm{~V}$ in this case) are responsible for the presence of a $50 \mathrm{~Hz}$ circulating current (29.84 A, Table 5), which causes the powers delivered by the upper and lower arms to be different. This can be seen in the vec- 
tor diagram in Figure 12. According to Equation (17), the powers in the two arms are $P_{u(l)}=v_{u(l) r m s} i_{u(l) x r m s} ;$ as the voltages $v_{u(l) r m s}$ are approximately equal, the difference between the powers is due to the differences between the currents $i_{u(l) x r m s}$ which, as can be seen in Figure 12, are due to the presence of the circulating current, which added to the currents $\pm \frac{i}{2}$ causes the currents $i_{u(l) x r m s}$ to have the appropriate value.

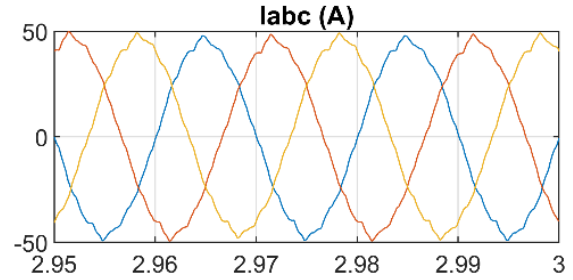

(a)

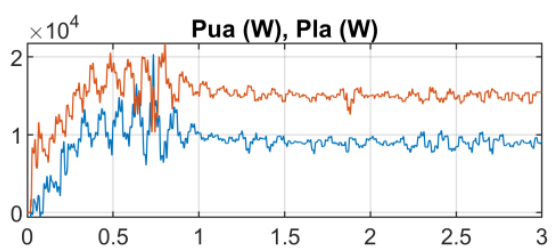

(b)

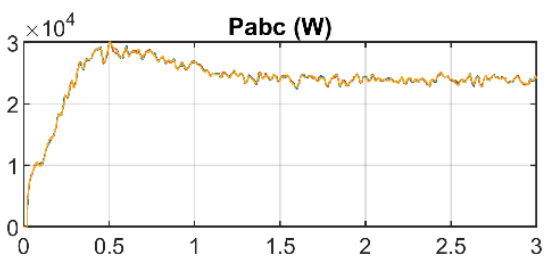

(c)

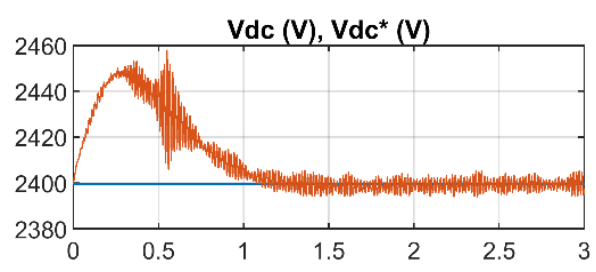

(d)

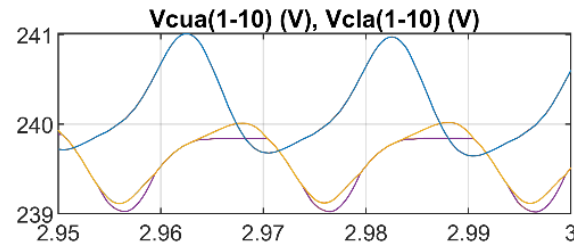

(e)

Figure 11. Simulation plots when the generation has an intermediate imbalance between arms. (a) Output currents. (b) Power of the upper and lower arms. (c) Power delivered by each phase. (d) Voltage on the DC link. (e) Capacitor voltages.

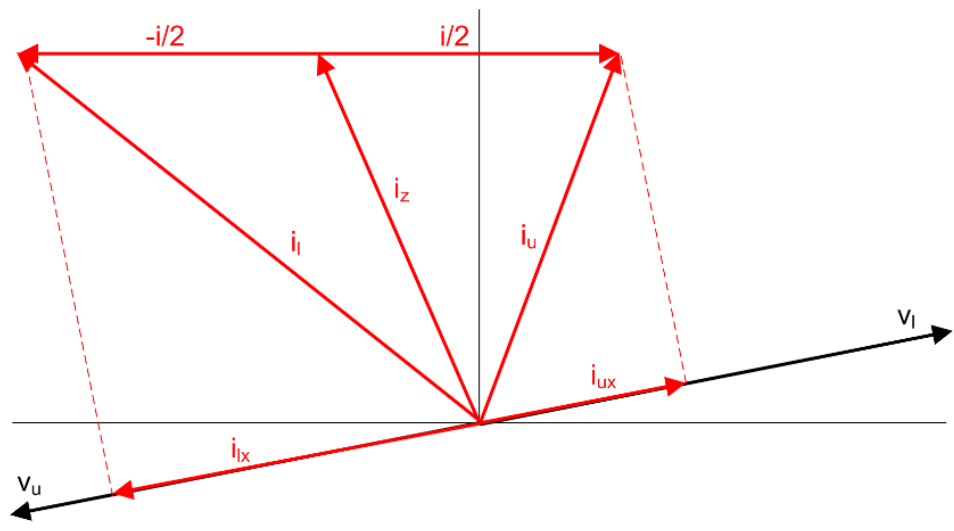

Figure 12. Vector diagram of the variables involved in the transmission of power from the arms when the generation has a medium imbalance between arms. All of them are $50 \mathrm{~Hz}$ variables (harmonic 1).

\subsubsection{Large Imbalance between Arms}

In this last sub-case, the power supplied by each phase is the same, $p_{W T a}=p_{W T b}=$ $p_{W T c}=18 \mathrm{~kW}$, but the power generated in the upper and lower arms of each phase has a large imbalance, $p_{u i}=3 \mathrm{~kW}, p_{l i}=15 \mathrm{~kW}$. For this, all generators supply $1500 \mathrm{~W}$, except for 8 generators in each upper arm which generate zero power. A case could have been simulated in which the 10 generators of the upper arm would deliver the same power 
of $300 \mathrm{~W}$, and the total power generated in the upper arm would be the same, but the preference has been to simulate a more unfavorable case in which modules that produce power and modules those that do not produce power must be kept in balance.

Figure 13 shows the simulation results. The MMC output currents $i_{a b c}$ (Figure 13a) are correct. The power of the upper arm of the first phase $p_{u a}$ is $3 \mathrm{~kW}$ and that of the lower $\operatorname{arm} p_{l a}$ is $15 \mathrm{~kW}$ (Figure 13b). The sum of both is the AC output power of the first phase $p_{a}$; the rest of phases have the same upper and lower arm and AC output power (Figure 13c). The DC link voltage (Figure 13d) has a small peak of $40 \mathrm{~V}$ during start-up and a slightly higher ripple than in the previous cases, but still small.

The $50 \mathrm{~Hz}$ circulating current has a high value, $50.08 \mathrm{~A}$ according to Table 5 , to enable the upper arm to deliver only $3 \mathrm{~kW}$ and the lower arm to deliver $15 \mathrm{~kW}$, as explained theoretically in Section 4.3. This has been illustrated in Figure 14, where a vector diagram including the $50 \mathrm{~Hz}$ voltages $v_{u(l)}$ and currents $i_{u(l)}$ of the arms is shown. According to Equation (17), the power delivered by each arm $P_{u(l)}$ can be calculated as the product $v_{u(l) r m s} i_{u(l) x r m s}$. It can be seen that $i_{u x}$ is much smaller than $i_{l x}$ to get $P_{u}$ to be much smaller than $P_{l}$, as is the case here. For this, $i_{z}$ takes an appropriate value to obtain, according to Equation (11), the values of $i_{u(l)}$ needed. The $i_{z}$ current is produced due to small differences between $v_{u}$ and $v_{l}$, which produce small values of $v_{2 L}$ according to Equation (15) and a circulating current of $50 \mathrm{~Hz}$ according to Equation (14). The small voltage differences between $v_{u}$ and $v_{l}$ are due to a small difference in the average voltages of the upper $(\approx 239.25 \mathrm{~V})$ and lower $(\approx 240.75 \mathrm{~V})$ capacitors (Figure $13 \mathrm{e})$. Otherwise, the $100 \mathrm{~Hz}$ component of $i_{z}$ is $4.27 \mathrm{~A}$ (Table 5), similar to the other cases.

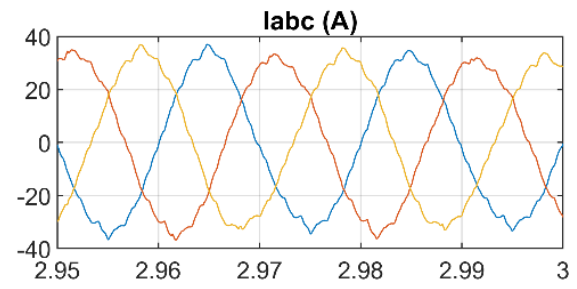

(a)

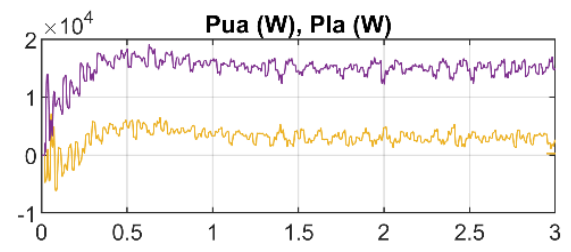

(b)

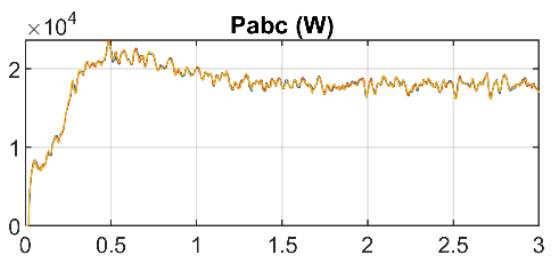

(c)

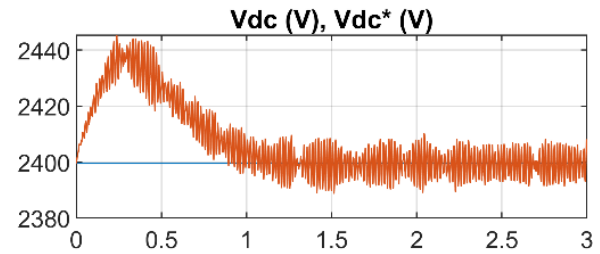

(d)

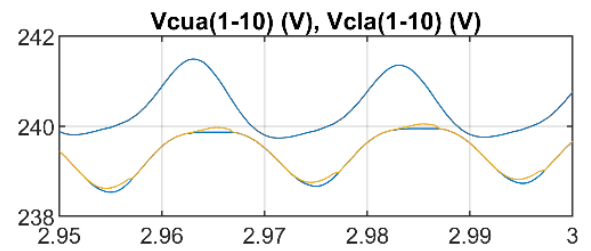

(e)

Figure 13. Simulation plots when the generation has a large imbalance between arms. (a) Output currents. (b) Power of the upper and lower arms. (c) Power delivered by each phase. (d) Voltage on the DC link. (e) Capacitor voltages. 


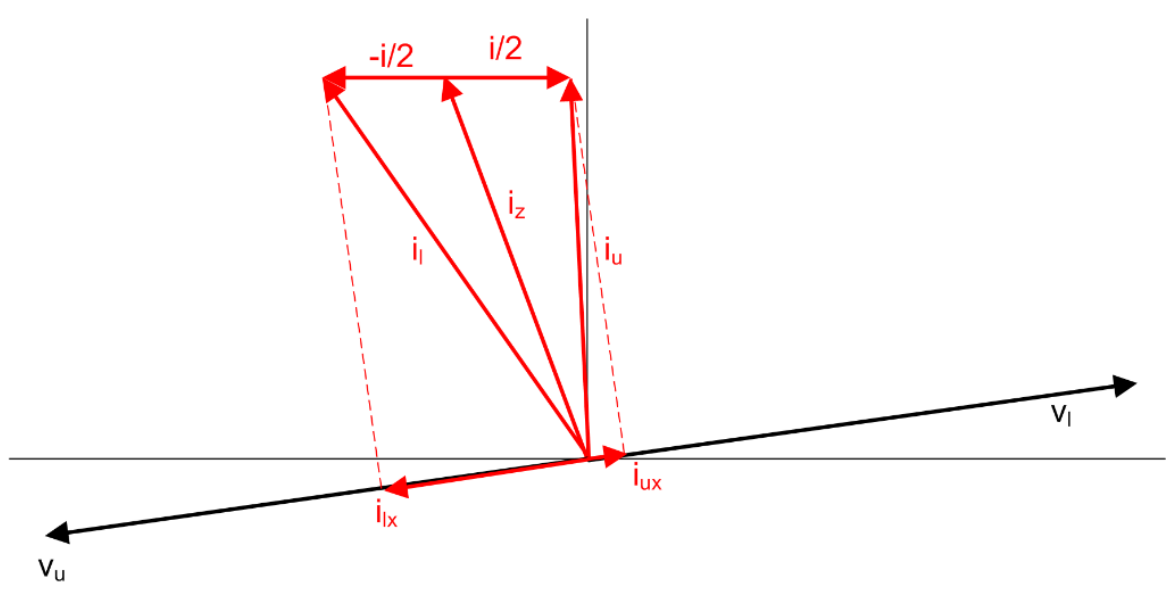

Figure 14. Vector diagram of the variables involved in the transmission of power from the arms when the generation has a large imbalance between arms. All of them are $50 \mathrm{~Hz}$ variables (harmonic 1).

\subsection{Conclusions of the Case Study}

Through the study of the possibilities that can happen in the generators, it has been demonstrated that a good operation of this topology can be obtained when the NLC modulation and the indicated control system are used. It has been shown that in all cases, the MMC AC voltages $v_{o a b c}$ and currents $i_{a b c}$, as well as the DC link voltage $v_{D C}$, take reasonable values. When all generators supply the same power, the system is balanced with low/null values of the DC and $50 \mathrm{~Hz}$ circulating currents, with only small circulating currents of $100 \mathrm{~Hz}$ which are usual in MMCs. When there are generation imbalances between phases, these are automatically balanced by the action of the DC circulating currents, while when the generation imbalances occur between arms, it is the $50 \mathrm{~Hz}$ circulating current that is responsible for establishing the balance.

It should be noted that the upper and lower arms carry a current, $i_{u}$ and $i_{l}$, which, according to Equation (11), includes half of the output current as well as the circulating current.

The results obtained in this paper regarding the imbalance of power generation have been compared with those obtained in [16,20], although both use PWM modulation whereas NLC is used in this paper; no previous works that use NLC have been found to establish a comparison. As a general comment, it should be noted that NLC is more appropriate than PWM when the number of generators is high.

Several renewable sources included in the SMs of the MMC are used in [20], and several PV generators are used in [16] connected through a DC/DC converter to each SM of the MMC. Both papers use circulating current regulators which, in order to send balanced power to the grid, transfer power between the arms or phases when imbalances occur between the generation of the arms or phases. In both papers, as well as in this paper, the imbalances between arms are corrected by the $50 \mathrm{~Hz}$ component of the circulating current and the imbalances between the phases by the DC circulating current. The fundamental difference between the work presented in this paper and those presented in $[16,20]$ is that, to control these processes, the latter two use a regulator for the circulating current and this paper does not use a regulator. However, in both $[16,20]$ as well as in this paper, power is transferred between arms and phases to send a balanced power to the grid.

According to the Technical Report IEC 61000-3-6, the maximum THD for coupling to the medium voltage grid is $6.5 \%$. The output voltage is almost the same in all cases tested, with THD in the range of $9.91-10 \%$. Taking into account the filtering effect carried out downstream of the converter by the filter and the coupling transformer, it will be easy to attain a THD below the limit set by the standards. Another interesting standard in this field is the IEC 61400-21 concerning to the measurement and assessment of electrical characteristics of wind turbines. Particularly interesting is the section devoted to fault ride-through (FRT). 


\section{Conclusions}

A new configuration for large wind farms made up of small- or medium-sized wind generators that includes them in the switching modules of an MMC has been presented.

The output waveform is obtained by NLC modulation, so the switching power losses are lower than in the case of PWM.

The developed setup achieves a large reduction in the number of semiconductors required compared to the normal configuration of a wind farm, decreasing from 12 IGBTs per wind generator to only 8 IGBTs. It also means a great reduction in the output voltage filtering requirements, since in the classical configuration a filter is needed at the output of the converter of each wind generator, while in the proposed configuration the output of the single converter could be connected directly to the single output transformer of the wind farm, or, at most, using a small filter.

The control features one outer loop for the MMC DC voltage and another one for the reactive power, as well as inner loops for the converter current control. This is followed by an NLC modulator and finally an algorithm that balances the capacitor voltages of the same arm.

The proposed topology, along with the control used, allows sending the power from the wind generators to the grid in a balanced way even in the case of generation imbalances between phases and/or between arms. To prove that this is possible, a case has been designed with specific parameters and tested in six very different situations with large imbalances. In all cases, the system behaved correctly.

Control systems for balancing switching modules including battery storage and photovoltaic generation can be found in the literature. In the proposed system, no control systems have been used for this function, but it has been found that the converter can balance itself naturally. Phase-to-phase imbalances are compensated by the presence of a DC circulating current that transfers power from the phases that generate more power to the phase that generates less power. Inter-arm imbalances are compensated by a $50 \mathrm{~Hz}$ circulating current caused by the occurrence of a small imbalance between the average values of the voltages of the upper and lower arms.

All this has been verified by the Matlab/Simulink simulation of one case and testing of many wind generation circumstances.

Author Contributions: Conceptualization, F.M.-R. and D.R.; methodology, F.M.-R., L.C.H.-d.L. and S.d.P.; software, F.M.-R. and D.R.; validation, D.R., L.C.H.-d.L. and S.d.P.; formal analysis, F.M.-R. and D.R.; investigation, F.M.-R., L.C.H.-d.L. and S.d.P.; resources, F.M.-R. and D.R.; data curation, D.R., L.C.H.-d.L. and S.d.P.; writing — original draft preparation, F.M.-R. and D.R.; writing-review and editing, L.C.H.-d.L. and S.d.P.; visualization, L.C.H.-d.L. and S.d.P.; supervision, F.M.-R. and D.R.; project administration, F.M.-R. and D.R. All authors have read and agreed to the published version of the manuscript.

Funding: This research received no external funding.

Conflicts of Interest: The authors declare no conflict of interest.

\section{References}

1. Friedrich, K. Modern HVDC PLUS application of VSC in Modular Multilevel Converter topology. In Proceedings of the 2010 IEEE International Symposium on Industrial Electronics, Bari, Italy, 4-7 July 2010.

2. Mahimkar, N.; Persson, G.; Westerlind, C. HVDC Technology for Large Scale Offshore Wind Connections. In Proceedings of the Smartelec, Vadodara, India, 16 April 2013; pp. 1-5.

3. Ingemansson, D.; Wheeler, J.D.; MacLeod, N.M.; Gallon, F.; Ruiton, O. The South — West Scheme: A New HVAC and HVDC Transmission System in Sweden. In Proceedings of the 10th IET International Conference on AC and DC Power Transmission (ACDC 2012), Birmingham, UK, 4-5 December 2012.

4. Du, S.; Liu, J. A study on dc voltage control for chopper-cell-based modular multilevel converters in d-statcom application. IEEE Trans. Power Deliv. 2013, 28, 2030-2038. [CrossRef]

5. Mohammadi, H.P.; Bina, M.T. A transformerless medium-voltage STATCOM topology based on extended modular multilevel converters. IEEE Trans. Power Electron. 2011, 26, 1534-1545. [CrossRef] 
6. Gangui, Y.; Jigang, L.; Gang, M.; Yu, L.; Yang, L.; Wei, S. Research on Modular Multilevel Converter Suitable for Direct-drive Wind Power System. Energy Procedia 2012, 17, 1497-1506. [CrossRef]

7. Engleitner, R.; Nied, A.; Cavalca, M.S.M.; Da Costa, J.P. Dynamic Analysis of Small Wind Turbines Frequency Support Capability in a Low-Power Wind-Diesel Microgrid. IEEE Trans. Ind. Appl. 2018, 54, 102-111. [CrossRef]

8. Yenduri, K.; Sensarma, P. Maximum Power Point Tracking of Variable Speed Wind Turbines with Flexible Shaft. IEEE Trans. Sustain. Energy 2016, 7, 956-965. [CrossRef]

9. Radwan, A.A.A.; Mohamed, Y.A.I. Grid-Connected Wind-Solar Cogeneration Using Back-to-Back Voltage-Source Converters. IEEE Trans. Sustain. Energy 2020, 11, 315-325. [CrossRef]

10. Rohner, S.; Bernet, S.; Hiller, M.; Sommer, R. Modulation, losses, and semiconductor requirements of modular multilevel converters. IEEE Trans. Ind. Electron. 2010, 57, 2633-2642. [CrossRef]

11. Martinez-Rodrigo, F.; Herrero-de Lucas, L.C.; de Pablo, S.; Rey-Boué, A.B.; Ramirez, D. Calculation of the number of modules and the switching frequency of a modular multilevel converter using near level control. Electr. Power Syst. Res. 2018, 165, 68-83. [CrossRef]

12. Soong, T.; Lehn, P.W. Assessment of Fault Tolerance in Modular Multilevel Converters with Integrated Energy Storage. IEEE Trans. Power Electron. 2016, 31, 4085-4095. [CrossRef]

13. Vasiladiotis, M.; Rufer, A. Analysis and control of modular multilevel converters with integrated battery energy storage. IEEE Trans. Power Electron. 2015, 30, 163-175. [CrossRef]

14. Soong, T.; Lehn, P.W. Evaluation of emerging modular multilevel converters for BESS applications. IEEE Trans. Power Deliv. 2014, 29, 2086-2094. [CrossRef]

15. Mao, M.; Ding, Y.; Chang, L.; Hatziargyriou, N.D.; Chen, Q.; Tao, T.; Li, Y. Multi-objective power management for EV fleet with MMC-based integration into smart grid. IEEE Trans. Smart Grid 2019, 10, 1428-1439. [CrossRef]

16. Bayat, H.; Yazdani, A. A Power Mismatch Elimination Strategy for an MMC-Based Photovoltaic System. IEEE Trans. Energy Convers. 2018, 33, 1519-1528. [CrossRef]

17. Rivera, S.; Wu, B.; Lizana, R.; Kouro, S.; Perez, M.; Rodriguez, J. Modular multilevel converter for large-scale multistring photovoltaic energy conversion system. In Proceedings of the 2013 IEEE Energy Conversion Congress and Exposition, ECCE 2013, Denver, CO, USA, 15-19 September 2013.

18. Rong, F.; Gong, X.; Huang, S. A Novel Grid-Connected PV System Based on MMC to Get the Maximum Power under Partial Shading Conditions. IEEE Trans. Power Electron. 2017, 32, 4320-4333. [CrossRef]

19. Bayat, H.; Yazdani, A. A Hybrid MMC-Based Photovoltaic and Battery Energy Storage System. IEEE Power Energy Technol. Syst. J. 2019, 6, 32-40. [CrossRef]

20. Soong, T.; Lehn, P.W. Internal power flow of a modular multilevel converter with distributed energy resources. IEEE J. Emerg. Sel. Top. Power Electron. 2014, 2, 1127-1138. [CrossRef]

21. Martinez-Rodrigo, F.; Ramirez, D.; Rey-Boue, A.B.; de Pablo, S.; Herrero-de Lucas, L.C. Modular Multilevel Converters: Control and Applications. Energies 2017, 10, 1709. [CrossRef]

22. Peralta, J.; Saad, H.; Dennetière, S.; Mahseredjian, J.; Nguefeu, S. Detailed and averaged models for a 401-level MMC-HVDC system. IEEE Trans. Power Deliv. 2012, 27, 1501-1508. [CrossRef]

23. Tu, Q.; Xu, Z. Impact of sampling frequency on harmonic distortion for modular multilevel converter. IEEE Trans. Power Deliv. 2011, 26, 298-306. [CrossRef]

24. Meshram, P.M.; Borghate, V.B. A simplified nearest level control (NLC) voltage balancing method for modular multilevel converter (MMC). IEEE Trans. Power Electron. 2015, 30, 450-462. [CrossRef]

25. Blaabjerg, F.; Teodorescu, R.; Liserre, M.; Timbus, A.V. Overview of control and grid synchronization for distributed power generation systems. IEEE Trans. Ind. Electron. 2006, 53, 1398-1409. [CrossRef]

26. Saeedifard, M.; Iravani, R. Dynamic performance of a modular multilevel back-to-back HVDC system. IEEE Trans. Power Deliv. 2010, 25, 2903-2912. [CrossRef]

27. Arruda, L.N.; Silva, S.M.; Filho, B.J.C. PLL structures for utility connected systems. In Proceedings of the Conference Record-IAS Annual Meeting (IEEE Industry Applications Society), Chicago, IL, USA, 30 September-4 October 2001. 